Este é um artigo publicado em acesso aberto sob uma licença Creative Commons https://creativecommons.org/licenses/by-nc/4.0/

\title{
A formação em regime de ciclos do Bacharelado Interdisciplinar em Saúde da Universidade Federal da Bahia e a proposta de Educação Interprofissional
}

\author{
Renata Meira Veras ${ }^{1}$ \\ Maria Thereza Ávila Dantas Coelho \\ Carmen Fontes Teixeira ${ }^{3}$ \\ Martha Azucena Traverso-Yépez ${ }^{4}$
}

Resumo: O sistema educacional superior na área da saúde vem sendo desafiado constantemente pelas mudanças contemporâneas pautadas pelas novas formas de organização e operacionalização da atenção integral à saúde da população. Este estudo tem como objetivo analisar e discutir alternativas à formação acadêmica em saúde, tomando como ponto de partida a experiência de implantação parcial da modalidade do regime de ciclos, na Universidade Federal da Bahia. Com base nas culturas humanísticas, artísticas e científicas, o Bacharelado Interdisciplinar em Saúde (BIS) oferece uma formação interdisciplinar de primeiro ciclo, que possibilita ao estudante situar-se frente à configuração atual do mercado de trabalho para as distintas profissões da área, bem como desenvolver competências e habilidades necessárias à atenção integral em saúde. O BIS, pautado numa postura colaborativa, prepara o estudante para o trabalho em equipe interdisciplinar. Também discute a proposta da educação interprofissional (EIP) como uma opção mais efetiva de formação para o cuidado integral em saúde, no segundo ciclo da formação. almejado pela EIP. Como continuidade para os cursos de segundo ciclo, sugere-se a inclusão da EIP na formação em saúde.

Palavras-chave: Educação Superior. Desenvolvimento de pessoal. Saúde.

The cycle system training in the Interdisciplinary Bachelor of Health at the Universidade Federal da Bahia and the Interprofessional Education proposal

Abstract: The higher education system in the health sector is been constantly challenged these days by contemporary changes arising from new forms of organization and operationalization of a comprehensive health care approach to people's health. This paper aims to analyze and discuss alternatives to academic training in health, starting with the experience of the partial implementation of the cycle system modality at the Universidade Federal da Bahia. It clarifies how, based on humanistic, aesthetic, and scientific principles, the Interdisciplinary Bachelor of Health (BIS) offers an interdisciplinary training during the first cycle, which enables students to become better prepared for the current configuration of the labor market in the different health sectors. We argue that this collaborative approach also helps students to develop competencies and skills necessary for a better integrated, comprehensive health care, preparing them to work in interdisciplinary teams. We also discuss the proposal of Interprofessional Education (IPE) as a more effective option during the second cycle of training, suggesting its inclusion at this stage as a logical follow-up.

Key words: Higher Education. Human resources development. Health. 
A formação em regime de ciclos do Bacharelado Interdisciplinar em Saúde da Universidade Federal da Bahia e a proposta de Educação Interprofissional

\section{Introdução}

A formação dos profissionais de saúde no Brasil vem sendo objeto de análise e reflexão nas últimas décadas do século XX e na primeira década do século XXI, tendo como principal preocupação a introdução de mudanças no processo de ensino-aprendizagem. Essas mudanças almejam adequar os perfis profissionais às necessidades e demandas oriundas das transformações que vêm ocorrendo no mercado de trabalho no setor, principalmente a partir da implementação do Sistema Único de Saúde (SUS) e da conformação, em paralelo, do Sistema de Assistência Médica Supletiva (SAMS) (PINTO et al., 2013; TEIXEIRA, 2013; ROCHA et al., 2016).

Nesse sentido, reconhece-se que a incorporação tecnológica ao processo de trabalho em saúde, bem como as mudanças que vêm ocorrendo na organização dos serviços, tanto no âmbito do sistema público, quanto no setor privado, têm fortalecido a tendência à divisão técnica e social do trabalho, com estimulo à especialização e subespecialização das atividades. Paralelamente, a incorporação de mecanismos gerenciais favorece a organização do trabalho em equipe, em todos os níveis, seja na atenção básica, seja nos serviços de média e alta complexidade (PEDUZI, 2003; 2013). Esse processo tem gerado uma reflexão sobre as políticas, modelos e práticas de formação de pessoal em saúde no país, chamando a atenção, inclusive, para a necessidade de introdução de mudanças em vários níveis (ALMEIDA FILHO, 2011; SANTOS; ALMEIDA FILHO, 2012).

Nessa perspectiva, este estudo tem como objetivo analisar e discutir alternativas à formação acadêmica em saúde, tomando como ponto de partida a experiência de implantação da modalidade do regime de ciclos, na Universidade Federal da Bahia. Apresenta-se, em seguida, a proposta de introdução da educação interprofissional (EIP) como uma alternativa mais efetiva de formação para o cuidado integral em saúde, no segundo ciclo da formação.

\section{As demandas da atual formação na área da saúde}

O sistema educacional está sendo desafiado a formar profissionais capacitados para trabalhar em equipe, aptos para prestar atendimento clínico integral e resolutivo, com competência ainda nos atos de gestão, prevenção e promoção da saúde (ALMEIDA FILHO et al., 2014). Entretanto, constata-se que o ensino superior, tanto nas universidades públicas quando nas instituições da rede privada, tende a reproduzir um modelo de formação de pessoal em saúde que privilegia o oferecimento de cursos profissionalizantes, sob responsabilidade de escolas e faculdades específicas, organizadas em departamentos que reproduzem a 
fragmentação do conhecimento em disciplinas sem articulação entre si. Esse modelo acaba gerando desenhos curriculares lineares, que periodicamente são submetidos a "reformas" que não alteram substancialmente a racionalidade que preside o processo de ensino, focado na memorização de conteúdos e aprendizagem acrítica das práticas que são realizadas nos serviços de saúde.

Assim, a formação acadêmica na área de saúde tende a reproduzir e fortalecer um modelo biomédico, assistencial e hospitalocêntrico, fundamentado basicamente na prática clínica, no atendimento individual, fortemente dependente do uso de tecnologias diagnósticas e terapêuticas que concorrem para a intensificação do processo de "medicalização" da saúde (ILLICH, 1975; NOGUEIRA, 2003) e "mercantilização" do processo de prestação de serviços (COHN; ELIAS, 2003; GADELHA, 2003; GADELHA; COSTA, 2012) no contexto do desenvolvimento do capitalismo brasileiro. Isso acarreta a perda da racionalidade integradora, a dificuldade em se desenvolver um pensamento que dê conta da complexidade dos determinantes sociais do processo saúde-doença e dos modos como este se apresenta nos diversos grupos da população e em indivíduos singulares. Esse modelo ainda tende a dificultar a compreensão dos desafios que se colocam na contemporaneidade para a organização e operacionalização de uma atenção integral à saúde da população (BATISTA, 2012) e ao cuidado humanizado que deve ser oferecido a cada pessoa que procura os serviços de saúde (PEDUZZI, 2003; MATTOS, 2008; AYRES, 2009).

Nesse cenário, portanto, coloca-se como uma necessidade e desafio a introdução de mudanças na educação superior em saúde, que transcendam os limites das reformas curriculares e proponham novos modelos de formação de pessoal. Esses novos modelos, necessariamente, incidirão sobre a organização e a gestão das atividades acadêmicas no âmbito das universidades. Nessa perspectiva, algumas universidades brasileiras passaram a experimentar mudanças administrativas e pedagógicas radicais, oferecendo opções de formação superior diversificada e inovadora, propiciando interlocução entre os mais diversos campos do conhecimento (ALMEIDA FILHO et al., 2015).

\section{A formação no regime de ciclos na UFBA e a EIP}

A educação deve ser responsável pela formação de um profissional capaz de agir e transformar sua prática vivenciada, tendo a instituição de ensino um papel fundamental para esta transformação (ALMEIDA FILHO et al., 2015). Assim, na área da saúde, a instituição de ensino deve se preocupar com a construção do conhecimento baseada em uma educação 
A formação em regime de ciclos do Bacharelado Interdisciplinar em Saúde da Universidade Federal da Bahia e a proposta de Educação Interprofissional

compartilhada de saberes entre profissionais e comunidade, por meio de um processo participativo que permita uma reflexão crítica da realidade e dos fatores determinantes de um viver saudável (AMORETTI, 2005).

No âmbito das formações em saúde, observa-se o desencadeamento de reformas curriculares estimuladas pela aprovação da atual Lei de Diretrizes e Bases para a Educação, que confere autonomia às instituições de ensino superior para adequar seus currículos às realidades regionais e às vocações das escolas, substituindo o antigo "currículo mínimo" pelas Diretrizes Curriculares Nacionais (DCN) (BRASIL, 1996). Outro caminho aberto em 2007, através do Programa de Apoio a Planos de Reestruturação e Expansão das Universidades Federais (REUNI), foi o de criação de novas modalidades de graduação, dentre as quais os bacharelados interdisciplinares (BRASIL, 2007). Um dos exemplos desse processo, inovador, é o modelo de ciclos desenvolvido na Universidade Federal da Bahia, que tem o Bacharelado Interdisciplinar em Saúde (BIS) como primeiro ciclo de formação, os cursos profissionais de progressão linear como segundo, e o mestrado e doutorado como terceiro ciclo (ALMEIDA FILHO et al., 2015). Nesse modelo, o BIS configura-se como porta de entrada ao processo de formação em saúde, para aqueles que optam por este curso no primeiro ciclo, contemplando conhecimentos gerais que qualificam os estudantes a situar-se criticamente no campo da saúde, ao tempo em que promove a ampliação da vivência na instituição universitária, agregando ao itinerário formativo conhecimentos e práticas de diversas áreas das artes, das humanidades e das ciências (TEIXEIRA; COELHO; ROCHA, 2013).

Ao lado dessas mudanças, existe o modelo de Educação Interprofissional (EIP) em Saúde, utilizado em alguns cursos profissionais, que se apresenta atualmente como a principal estratégia para formar profissionais aptos para o trabalho em equipe, prática essencial para o cuidado integral em saúde (BATISTA, 2012). A EIP é conceituada como o aprendizado que acontece quando duas ou mais profissões aprendem sobre, com e entre si, de forma a melhorar a colaboração e os resultados na saúde (WHO, 2010). O reconhecimento da necessidade desse modelo de formação tem levado a mudanças na educação profissional em saúde em muitos países.

O Bacharelado Interdisciplinar em Saúde (BIS) foi planejado, então, para superar os desafios da formação de recursos humanos no campo da saúde. Com efeito, o BIS pode ser definido como um curso universitário de formação geral e de natureza interdisciplinar. Possui terminalidade própria, podendo ser caracterizado como primeira etapa para o ingresso em cursos profissionais de progressão linear, ou para a formação acadêmica de pós-graduação humanística, científica ou artística, ou, ainda, para o ingresso no mercado de trabalho, em 
ocupações que não exijam formação profissional específica (ROCHA et al., 2014). O Instituto de Humanidades, Artes e Ciências Prof. Milton Santos (IHAC) foi criado para abrigar esse curso, dentre os demais Bacharelados Interdisciplinares da Universidade Federal da Bahia, em Humanidades, Artes, Ciência e Tecnologia.

Portanto, o BIS se caracteriza como o primeiro ciclo do processo de formação superior em saúde, para os alunos que optam por esse regime de ciclos, em caráter interdisciplinar, propedêutico, como etapa prévia, caso deseje o estudante, ao segundo ciclo (constituído pelos cursos profissionais voltados para o desenvolvimento de competências e habilidades requeridas para o ingresso no mercado de trabalho das diversas profissões) ou ao terceiro, formado pelos cursos de mestrado (profissionais e acadêmicos) e doutorado, como observamos no gráfico 1 abaixo:

\section{Gráfico 1 - O modelo de ciclos}

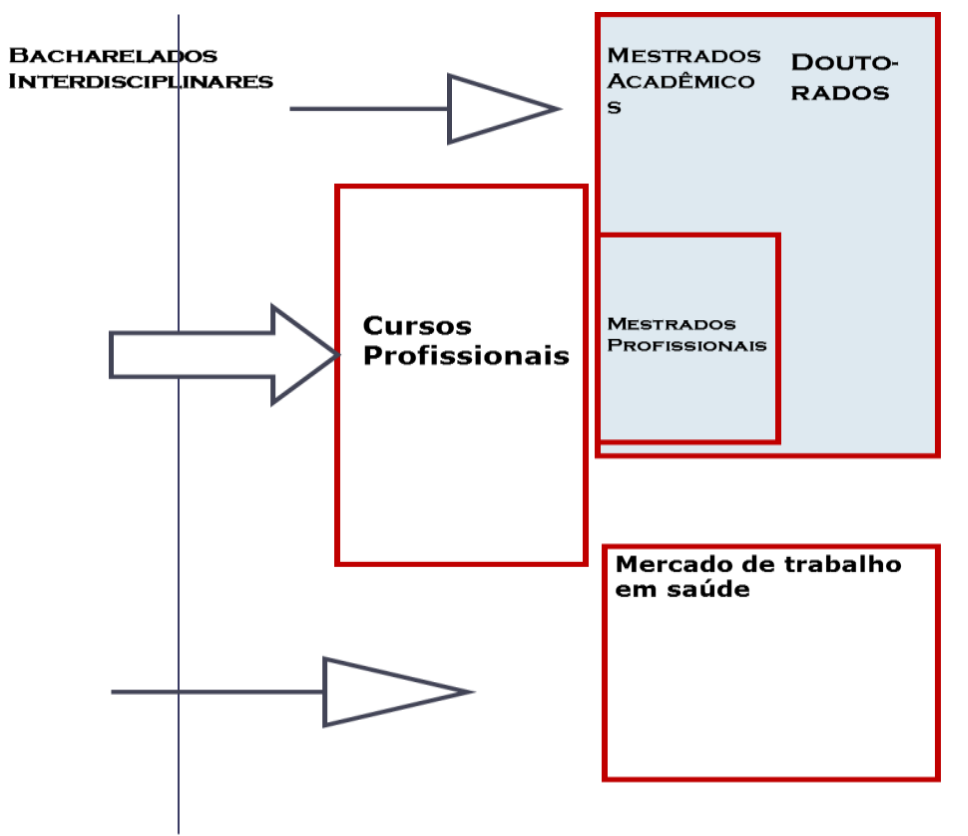

Fonte: UNIVERSIDADE FEDERAL DA BAHIA (UFBA). Bacharelados Interdisciplinares: projeto pedagógico. Salvador, 2008. (com adaptação)

De acordo com o projeto pedagógico do BIS, este é

um curso de graduação de duração plena que visa agregar uma formação geral humanística, científica e artística ao aprofundamento no campo da saúde, promovendo o desenvolvimento de competências e habilidades que conferem autonomia para a aprendizagem e uma inserção mais abrangente e multidimensional na vida social (UFBA, 2010, p. 7).

O perfil do egresso é enfatizado no projeto pedagógico do curso através das competências gerais relacionadas com a capacidade de "aprender a aprender", competências 
A formação em regime de ciclos do Bacharelado Interdisciplinar em Saúde da Universidade Federal da Bahia e a proposta de Educação Interprofissional

cognitivas e habilidades específicas que permitem ao estudante compreender a complexidade do campo da saúde na contemporaneidade, acrescentando um conjunto de valores como a luta pelos direitos humanos, preservação do meio ambiente, a defesa dos princípios democráticos e a valorização da diversidade cultural, como podemos verificar no quadro 1 abaixo (UFBA, 2010; TEIXEIRA; COELHO, 2014).

\section{Quadro 1 - Perfil do egresso do BI Saúde}

\begin{tabular}{|c|c|}
\hline $\begin{array}{l}\text { Competências e } \\
\text { habilidades gerais }\end{array}$ & $\begin{array}{l}\text { - } \quad \text { Capacidade de abstração, análise e síntese de conhecimentos; } \\
\text { - Habilidades para buscar, processar e analisar informação procedente de fontes } \\
\text { diversas; } \\
\text { - } \quad \text { Capacidade de comunicação oral e escrita em língua portuguesa; } \\
\text { - Habilidades no uso das tecnologias da informação e da comunicação; }\end{array}$ \\
\hline $\begin{array}{l}\text { Competências e } \\
\text { habilidades } \\
\text { específicas }\end{array}$ & $\begin{array}{l}\text { - Compreender a complexidade do campo da saúde nas sociedades } \\
\text { contemporâneas; } \\
\text { - Identificar e analisar problemas de saúde no âmbito individual e coletivo; } \\
\text { - Analisar políticas públicas, programas e projetos da área da saúde; } \\
\text { - Identificar e analisar as tendências do mercado de trabalho e das práticas } \\
\text { profissionais em saúde; }\end{array}$ \\
\hline $\begin{array}{l}\text { Competências } \\
\text { valorativas e } \\
\text { compromissos } \\
\text { éticos }\end{array}$ & $\begin{array}{l}\text { - } \quad \text { Responsabilidade social e compromisso cidadão; } \\
\text { - } \quad \text { Valorização e respeito pela diversidade cultural; } \\
\text { - } \quad \text { Consolidação dos valores democráticos na sociedade contemporânea; } \\
\text { - } \quad \text { Preservação do meio ambiente; } \\
\text { - } \quad \text { Compromisso ético-político no campo da saúde. }\end{array}$ \\
\hline
\end{tabular}

Fonte: UNIVERSIDADE FEDERAL DA BAHIA (UFBA). Projeto pedagógico dos bacharelados interdisciplinares. Salvador, 2010.

Para Teixeira, Coelho e Rocha (2013), o BIS apresenta-se como um espaço de formação universitária de caráter introdutório ao campo da saúde, onde se enfatiza a apropriação de conhecimento e desenvolvimento de habilidades e valores que contemplam a compreensão da dimensão histórica e social das concepções e práticas de saúde, contribuindo, portanto, para o enriquecimento intelectual, a humanização e aprimoramento da formação profissional na área da saúde. Além disso, também possibilita ao estudante situar-se e sensibilizar-se com relação aos determinantes sociais da saúde, às propostas de enfrentamento político dos problemas de saúde e à configuração do mercado de trabalho para distintas profissões da área, de modo a determinar um fazer mais competente e atuante.

Com base nessa proposta geral, o projeto pedagógico do BIS contempla a organização de um conjunto de eixos ou módulos que agregam diversos componentes curriculares, cujos conteúdos programáticos abordam um conjunto de conhecimentos selecionados de modo a que os estudantes possam atingir os objetivos esperados. O Quadro 2 apresenta uma síntese desses módulos e seus respectivos componentes curriculares (CC), podendo-se constatar, pela simples 
leitura dos títulos, que estes materializam os pressupostos epistemológicos do curso, na medida em que contemplam o aprendizado de uma perspectiva interdisciplinar na abordagem de questões contemporâneas, bem como articulam a aproximação dos estudantes a conteúdos provenientes das culturas artística, humanística e científica, aos quais agregam-se conteúdos específicos do campo da saúde.

\section{Quadro 2 - Síntese da distribuição dos componentes curriculares do BIS}

\begin{tabular}{|c|c|c|}
\hline Eixo ou módulos & Componente curricular & Carga Horária \\
\hline Módulo interdisciplinar & $\begin{array}{l}\text { HACA01- Estudos sobre a Contemporaneidade I } \\
\text { HACA34 - Estudos sobre a Contemporaneidade II }\end{array}$ & $\begin{array}{l}68 h \\
68 h\end{array}$ \\
\hline Módulo Culturas & $\begin{array}{l}2 \text { CC Cultura humanística (a escolher) } \\
2 \text { CC Cultura artística (a escolher) }\end{array}$ & $\begin{array}{l}136 \mathrm{~h} \\
136 \mathrm{~h}\end{array}$ \\
\hline Eixo Linguagens & $\begin{array}{l}\text { LETE43 - Língua portuguesa, poder e diversidade } \\
\text { LETE45 - Leitura e Produção de textos em Língua Portuguesa } \\
\text { HACA78 - Oficina de textos acadêmicos e técnicos em saúde }\end{array}$ & $\begin{array}{l}68 \mathrm{~h} \\
68 \mathrm{~h} \\
68 \mathrm{~h}\end{array}$ \\
\hline $\begin{array}{l}\text { Formação específica } \\
\left(1^{\mathrm{a}} \text { etapa) }\right. \\
\text { CC obrigatórios }\end{array}$ & $\begin{array}{l}\text { HACA10 - Introdução ao Campo da saúde } \\
\text { HACA40 - Campo da Saúde: saberes e práticas } \\
\text { HACB17 - Saúde, Educação e Trabalho }\end{array}$ & $\begin{array}{l}68 h \\
68 h \\
68 h\end{array}$ \\
\hline $\begin{array}{l}\text { Formação específica } \\
\left(2^{\text {a }} \text { etapa }\right) \\
\text { CC optativos }\end{array}$ & $\begin{array}{l}11 \text { componentes curriculares oferecidos pelas unidades de saúde } \\
\text { e/ou pelo Instituto de Humanidades, Artes e Ciências }\end{array}$ & $748 \mathrm{~h}$ \\
\hline TOTAL & & $1020 \mathrm{~h}$ \\
\hline $\begin{array}{l}\text { Atividades } \\
\text { Complementares }\end{array}$ & & $360 \mathrm{~h}$ \\
\hline Componentes livres & (7 componentes livres de 68 horas) & $476 h$ \\
\hline TOTAL GERAL & & $2400 h$ \\
\hline
\end{tabular}

Fonte: UNIVERSIDADE FEDERAL DA BAHIA (UFBA). Projeto pedagógico dos bacharelados interdisciplinares. Salvador, 2010.

Os estudos sobre a contemporaneidade são realizados integralmente pelos docentes do IHAC, enquanto que dois dos componentes do eixo linguagens (LETE43 e LETE45) são ofertados pelo Instituto de Letras. O componente Oficina de Textos Acadêmicos e Técnicos em Saúde completa esse Eixo, discutindo as múltiplas formas de expressão e comunicação dos conhecimentos e tecnologias produzidos e difundidos na área da saúde. Através desse componente, os estudantes aprendem a ler e a analisar artigos científicos, resenhas, projetos, relatórios de pesquisa, documento institucionais, leis, portarias, planos, programas e projetos de intervenção no campo da saúde.

No Módulo Culturas, o estudante deve cursar pelo menos dois componentes classificados como humanísticos e dois como artísticos. Essa classificação se dá na medida em que se considera que os componentes curriculares da área das artes são considerados como "cultura artística", enquanto que os componentes relacionados à área da filosofia e ciências humanas são considerados como "cultura humanística". Teixeira e Coelho (2014) destacam a 
A formação em regime de ciclos do Bacharelado Interdisciplinar em Saúde da Universidade Federal da Bahia e a proposta de Educação Interprofissional

dificuldade em separar os componentes relacionados a essas duas culturas, uma vez que Almeida Filho (2005) discute o campo da saúde como sendo recortado por conhecimentos e práticas que ultrapassam os limites disciplinares, apresentando-se como multi e interdisciplinares, resistindo também a serem classificados como partes de uma determinada cultura, sendo, por natureza, multi e até transculturais.

Quanto à formação específica em saúde, Teixeira e Coelho (2014) destacam que os componentes curriculares desse eixo foram criados para darem conta de uma visão panorâmica do campo da saúde. As autoras salientam que o conceito de saúde é pouco explorado na literatura científica da área, enquanto que no BIS ele passa a ser revisitado do ponto de vista histórico, sociológico, antropológico, político e cultural.

A formação específica (11 componentes curriculares optativos em saúde), como também os sete componentes livres que devem ser escolhidos pelos estudantes do BIS entre o elenco de componentes oferecidos pelas unidades da UFBA, representam a possibilidade de conhecer e explorar aspectos diversos da grande área das ciências em saúde. Nesse eixo, os estudantes do BIS cursam os componentes curriculares juntamente com estudantes de outros cursos, tanto da área de saúde, como de outras áreas (no caso dos componentes livres). Para Almeida Filho (2014), o BIS representa uma possibilidade real de mudanças na formação em saúde, devido ao engajamento de sua proposta nos valores de humanização, participação e integralidade. A autonomia é trabalhada no BIS, uma vez que este estimula o estudante a desenhar seu próprio percurso acadêmico. O curso visa também um sistema de formação com base em métodos de trabalho, mais que em conteúdo, direcionados a habilitar o estudante à busca permanente de soluções, capacitando-o a continuar aprendendo durante sua futura formação e prática profissional.

\section{A Educação Interprofissional como alternativa de segundo ciclo}

A partir de 1988, a Organização Mundial de Saúde (OMS) passou a apoiar e a estimular a EIP, principalmente a partir de dois relatórios: Continuing Education for Physicians (WHO, 1988a) e Learning Together to Work Together for Health (WHO, 1988b). Em 2010, a OMS demonstrou seu compromisso com esse tipo de educação criando o Health Professionals Global Network (HPGN), a fim de maximizar o potencial de todos profissionais de saúde através de colaboração interprofissional, contribuindo dessa forma com a agenda de saúde global. Em paralelo, publicou o Framework for Action on Interprofessional Education and Collaborative Practice (WHO, 2010). Esse documento apontou a necessidade de EIP e de uma prática 
colaborativa para melhorar sistemas de saúde fragmentados, tanto em países desenvolvidos como também em subdesenvolvidos.

Os conceitos mais utilizados pelos autores sobre EIP, aprendizado interprofissional e colaboração interprofissional estão apresentados no Quadro 3 a seguir.

\section{Quadro 3: Definição de EIP e práticas colaborativas}

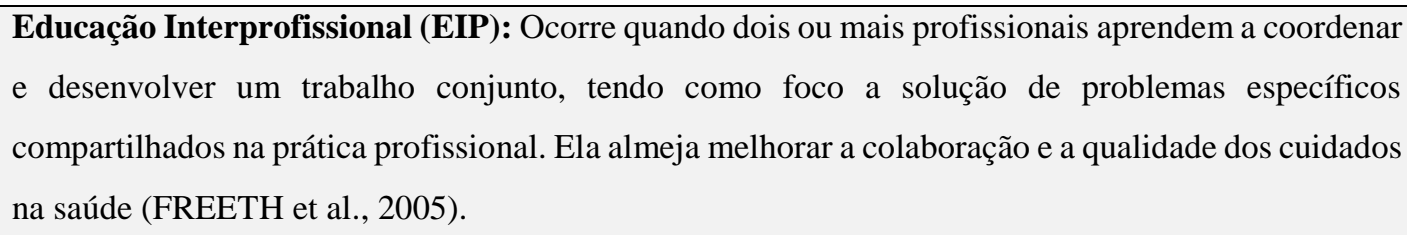

Aprendizagem Interprofissional (AIP): Aprendizado decorrente da interação entre membros (ou estudantes) de duas ou mais profissões. Este pode ser um produto da educação interprofissional ou pode acontecer espontaneamente em locais de trabalho ou espaços educacionais (FREETH et al., 2005).

Colaboração Interprofissional (CIP): É o processo de desenvolvimento e manutenção de trabalho interprofissional efetivo que relaciona aprendizes, praticantes, pacientes, clientes, familiares e comunidades para possibilitar melhores espaços de assistência à saúde (CIHC, 2009).

Fonte: Adaptado de THISTLETHWAITE, J. Interprofessional education: a review of context, learning and the research agenda. Medical Education, Vancouver, BC, v. 46, p. 58-70, 2012.

No campo da saúde, a interprofissionalidade pode ser compreendida como a resposta às realidades fragmentadas das práticas de assistência à saúde. Diferentes profissões têm sua origem a partir de diferentes disciplinas e de diferentes organizações de saúde, cada um possuindo seus próprios conteúdos e marcos conceituais, olhares específicos sobre os pacientes e suas necessidades, bem como uma maneira específica de atuar no complexo sistema de saúde. Assim, a interprofissionalidade surge da preocupação dos profissionais em reconhecer suas similaridades e seus pontos de vista divergentes, e isso envolve uma contínua interação e compartilhamento de conhecimentos entre profissionais para resolver ou explorar a variedade de questões educacionais e de saúde, a fim de melhorar a integralidade do atendimento e a participação do paciente (D’AMOUR; OANDANSAN, 2005).

Portanto, percebe-se que um dos objetivos da EIP é capacitar estudantes para adquirirem habilidades para trabalhar com diferentes profissionais de saúde, para entender seus papéis no sistema de saúde e para estarem preparados para uma atuação interprofissional. Além disso, diversos fatores têm precipitado o aumento do interesse na EIP: mudanças demográficas (em particular o envelhecimento populacional); aumento de morbidades que requerem cuidados 
A formação em regime de ciclos do Bacharelado Interdisciplinar em Saúde da Universidade Federal da Bahia e a proposta de Educação Interprofissional

complexos; novos modelos de atenção à saúde; avanços tecnológicos; aumento das especialidades médicas, entre outros. Alguns desses fatores estão bem explorados em um artigo publicado pela Lancet Comission em 2010, uma comissão de 20 profissionais e líderes acadêmicos focados em desenvolver uma visão compartilhada e estratégias para a educação de profissionais da saúde (FRENK et al., 2010). A comissão se refere a uma crise lenta na educação em saúde, causada pela fragmentação do currículo, utilizando o termo "trabalho em silo" para o tipo de trabalho fragmentado.

Os métodos da EIP podem variar de acordo com o estágio e os objetivos a que se destina. O quadro 4 sintetiza os sete tipos de métodos mais comuns. A combinação de dois ou mais métodos interativos de aprendizado pode contribuir para um aprendizado mais eficaz.

\section{Quadro 4: Métodos de EIP}

1. Aprendizado baseado em seminário

2. Aprendizado baseado em observação (shadowing)

3. Aprendizado baseado em problemas

4. Aprendizado baseado em simulação

5. Aprendizado baseado na prática clínica (experiential learning)

6. Aprendizado E-learning

7. Aprendizado misto (integrando e-learning com outro método tradicional)

Fonte: Adaptado de REEVES, Scott. Porque precisamos da educação interprofissional para um cuidado efetivo e seguro. Interface, São Paulo, v. 20, n. 56, p. 185-96, 2016.

Reeves (2016) e Freeth e colaboradores (2005) atentam para o fato de que, sendo a EIP eletiva ou voluntária para os estudantes (ou estudantes de alguns cursos), isto pode passar a mensagem de que este tipo de aprendizagem não tem grande valor para sua formação profissional, contribuindo para a redução do interesse do estudante. Receber apoio de líderes estudantis e profissionais, assim como incentivar a participação de profissionais atuantes também pode contribuir para o sucesso e adesão à EIP.

A literatura aponta ainda uma gama de modelos de EIP considerados como programas, módulos e espaços de aprendizagem. Esses variam entre 1 hora de sessão grupal com dois ou mais profissionais diferentes até um curso inteiro planejado com essas sessões grupais. Para Thistlethwaite (2012), as decisões sobre o tipo de modelo, quando e como implantá-lo dependem mais de questões pragmáticas e de logística do que do desenho pedagógico que se 
deseja. Langton (2009) sintetizou cinco tipos mais comuns de EIP, conforme o Quadro 5 abaixo:

\section{Quadro 5: Tipos de EIP}

1. Um ou mais módulos inseridos em um novo ou já existente currículo

2. Através da prática clínica

3. Um currículo comum para todas as formações em saúde

4. Educação virtual em paralelo com outros cursos

5. Focado num tópico de trabalho

Fonte: Langton, H. Interprofessional education in higher education institutions: models, pedagogies and realities. In: BLUTEAU, P.; JACKSON, A. (Eds.). Interprofessional education. Making it Happen. Basingstoke: Palgrave Macmillan, 2009. p. 37-58.

No Canadá há uma proposta de inserir os estudantes do campo da saúde nas práticas de uma educação interprofissional desde o início da formação acadêmica e continuar com reforços regulares nas diferentes etapas da formação (CURRAN; SHARPE, 2007). Observa-se que os tipos de EIP podem variar, podendo existir inclusive combinações deles. Os princípios de tais práticas focam-se principalmente nos problemas de saúde que demandam uma abordagem interprofissional para sua resolução (por exemplo saúde da criança, cuidado de idosos, cuidados da saúde mental etc.).

Como exemplo do tipo 3 de EIP no Brasil, a Faculdade de Ciências Médicas e da Saúde de Juiz de Fora (FCMS/JF) vem desenvolvendo-o através da realização do Programa Integrador, junto à Atenção Primária à Saúde, que tem como objetivo contribuir para a formação integrada de profissionais de saúde e para a melhoria do Sistema de Saúde do Município de Juiz de Fora. Esse programa é um dos componentes centrais da estrutura curricular dos cursos de Medicina, Enfermagem, Fisioterapia, Farmácia e Odontologia, visando a indissociabilidade entre teoria-prática, a integração da Faculdade ao meio social local e regional, bem como a integração entre os cursos. Trata-se de um eixo integrador comum a todas as formações em saúde supracitadas, que deve ser cursado pelos alunos (AGUILAR DA SILVA; SCAPIN; BATISTA, 2011). Igualmente, o Campus Baixada Santista da Universidade Federal de São Paulo, a Faculdade de Medicina de Marília e a Universidade Federal do Sul da Bahia vêm desenvolvendo um currículo integrado para os cursos da área de saúde, focando na EIP (SILVA, 2011; BATISTA, 2012; ALMEIDA FILHO et al., 2015). Esta última ainda adota o regime de ciclos, modelo inovador no Brasil, oferecendo o curso de Bacharelado Interdisciplinar em Saúde como primeira etapa de formação (pré-requisito) para os cursos da área de saúde. 
A formação em regime de ciclos do Bacharelado Interdisciplinar em Saúde da Universidade Federal da Bahia e a proposta de Educação Interprofissional

Além do tipo de modelo, outro debate presente na literatura refere-se a quando introduzir a EIP como programa educacional para profissionais da saúde. Autores como Horak e O`leary (1998) e Leaviss (2000) enfatizam a necessidade de incluir essa estratégia ainda na graduação, principalmente nos dois primeiros anos do curso. Para esses autores, introduzir a EIP ainda na graduação significa que o desenvolvimento de estereótipos e atitudes negativas em relação a outros profissionais de saúde (o que é difícil de ser revertido) pode não acontecer com esse tipo de formação nos primeiros anos de curso. Além disso, para Carpenter e Hewstone (1996), mesmo que esses construtos sejam desenvolvidos no início do curso, ao se introduzir a EIP ainda na graduação os estudantes poderão ter a oportunidade de desconstruí-los.

Por outro lado, autores como Petrie (1976), Parsell e Bligh (1998) advogam a aplicação da EIP entre profissionais já formados e trabalhando em unidades de saúde. Para esses autores, nessa etapa os indivíduos estarão mais seguros profissionalmente, já que possuem melhor compreensão da sua própria identidade profissional e do seu papel. Esse segundo modelo, nomeado como Educação Permanente em Saúde (EPS), já vem sendo utilizado no Brasil através de algumas estratégias do Ministério da Saúde. A EPS é reconhecida como trabalho que articula a atenção à saúde, a formação, a gestão e o controle social para a transformação das práticas de saúde e da organização do trabalho (MERHY; FEUERWERKER; CECCIM, 2006).

A EPS teve um grande destaque entre os anos de 2003 e 2005, no Brasil, quando foi criada a Secretaria da Gestão do Trabalho e da Educação na Saúde (SGTES). Entre 2005 e 2006 foram instalados 96 polos de EPS, contando com a participação de mais de 1.400 atores institucionais, profissionais da saúde, gestores e educadores. No entanto, Miccas e Batista (2014) relatam que a falta de continuidade nos investimentos, o pouco comprometimento de gestores, a dificuldade de construir uma dinâmica de trabalho ágil e disputas por poder resultaram em redução e constituição mais lenta dos espaços. Os fatores predominantes para o insucesso dos polos apontados pelas autoras foram: alta rotatividade dos profissionais nos setores, distribuição irregular regional, crescente especialização com dependência de tecnologias mais sofisticadas e predomínio da EPS na formação hospitalar, que resultaram em conceitos imprecisos de integralidade e promoção da saúde.

Durante a última década, alguns autores trataram de empreender estratégias para examinar e sumarizar a evidência no campo da EIP (COOPER et al., 2001; HAMMICK et al., 2007; REEVES et al., 2008; AGUILAR DA SILVA; SCAPIN; BATISTA, 2011; LAPKIN, LEVVETT-JONES; GILLIGAN, 2013). Nessas avaliações, dois instrumentos são descritos com maior frequência na literatura - um Questionário com Escala de Percepção da Experiência Interprofissional (IEPS), criado em 1990 e modificado em 2007 (LUECHT et al., 1990; 
MCFADYEN; MCLAREN; WEBSTER, 2007) e um outro questionário para avaliação de atitudes e prontidão (RIPLS), que utiliza uma escala Likert para avaliar a competência para o trabalho de equipe e colaboração, a identidade profissional e a discussão dos papéis profissionais (BATISTA, 2012).

\section{Considerações Finais}

A formação interdisciplinar na saúde pode oferecer possibilidades de impactos positivos em relação à qualidade da assistência à saúde prestada no serviço público. Ressaltamos que mais de $65 \%$ dos profissionais de saúde que se formam no Brasil atuam no Sistema Único de Saúde. Por conta disso, as Diretrizes Curriculares Nacionais reforçam a necessidade de orientar a formação dos profissionais para a inserção nesse sistema público, o que tem propiciado certo esforço por parte das instituições universitárias em aproximar o ensino-aprendizagem às necessidades do mesmo.

Assim, a crise no setor saúde vem sendo considerada como reflexo da crise no sistema educacional do país, que está em descompasso com as necessidades sociais da saúde da população. A questão-chave para os problemas de saúde no Brasil parece ser a deformação do ensino - humanístico e acadêmico - profissional da saúde (ALMEIDA FILHO, 2013, 2014). Sem dúvida, a resolução dos problemas no setor saúde parece exigir uma mudança na formação desses profissionais, valorizando as vertentes da humanização, da ética, da participação social e do trabalho em equipe.

Nesse sentido, percebe-se que a interdisciplinaridade na formação colabora mais para uma atuação interprofissional, que é resultado de competências baseadas no trabalho em equipe, discussão de papéis profissionais, compromisso na solução de problemas e negociação na tomada de decisão. Para tanto, é necessário que a educação pautada na interdisciplinaridade resgate, nos currículos, os conteúdos educativos das diversas culturas humanística, artística e científica, os métodos, os contextos culturais, as redes de comunicação, a distribuição dos espaços e tempos educativos, o planejamento pedagógico e didático, entre outros. Esse tipo de educação deve possibilitar métodos que ensinem a pensar, a pensar sobre o já pensado, aprendendo a aprender, uma educação que, superando a visão cartesiana da neutralidade e da objetividade do conhecimento, recupere os valores associados ao saber, colaborando com a formação de cidadãos e profissionais, com uma ética de vida que guie a sua atuação cotidiana, formando profissionais abertos ao outro, às diferenças, ao diálogo e ao desconhecido (LUZZI; PHILIPPI JR., 2011). 
A formação em regime de ciclos do Bacharelado Interdisciplinar em Saúde da Universidade Federal da Bahia e a proposta de Educação Interprofissional

O reconhecimento da necessidade da EIP em saúde tem levado a mudanças na educação profissional em saúde em muitos países. O Bacharelado Interdisciplinar em Saúde da Universidade Federal da Bahia tem uma proposta interdisciplinar que se antecipa e prepara o estudante para a abertura ao futuro trabalho interprofissional. Assim, torna-se um espaço privilegiado para que esse tipo de inovação na formação em saúde, no segundo ciclo, ocorra no Brasil. Esse é um desafio proposto em termos do ensino da prática interprofissional e colaborativa, tendo em vista a compreensão dos estudantes da saúde acerca dos papéis dos outros profissionais e a promoção de uma futura cooperação entre grupos de profissionais. A formação interdisciplinar ofertada pelo Bacharelado Interdisciplinar em Saúde tende a formar um novo perfil de bacharel em saúde, consoante com as diretrizes do SUS. O BIS está diretamente relacionado a um tipo de educação voltado para a interdisciplinaridade e para o trabalho em equipe. Como um primeiro ciclo de uma formação pautada em princípios, valores, métodos e práticas totalmente renovados, o BIS tem o potencial de empreender mudanças na formação em saúde, uma vez que sua estrutura possibilita um modo de fazer engajado nos princípios ético-políticos da equidade, solidariedade e colaboração.

A inclusão da EIP no currículo dos cursos de saúde de segundo ciclo, como um projeto de continuidade da proposta interdisciplinar na formação profissional, tende a derrubar barreiras, aumentar confiança, desconstruir estereótipos e melhorar as relações interprofissionais. “Aprender juntos para trabalhar juntos por uma saúde melhor” é uma citação que inicia o documento marco para a ação na educação interprofissional e práticas colaborativas, publicado em 2010 pela Organização Mundial da Saúde (OMS). Trilhar um segundo ciclo de formação profissional numa perspectiva apenas disciplinar, após a realização de uma trajetória interdisciplinar no primeiro ciclo, reduz as potencialidades de tal formação, assim como retira a chance de construção, durante a formação acadêmica profissional, de saberes e práticas calcadas no diálogo entre as profissões, necessário ao cuidado integral à saúde. Ao mesmo tempo, ter uma formação profissional na perspectiva da EIP tendo antes trilhado um primeiro ciclo de formação geral (a exemplo do Bacharelado Interdisciplinar em Saúde) possibilita maior abertura do corpo discente para a colaboração interprofissional e o trabalho em equipe, com conhecimentos já enriquecidos pelas culturas humanísticas, artísticas e científicas da primeira formação. 


\section{Referências}

AGUILAR DA SILVA, Rinaldo Henrique; SCAPIN, Luciana Teixeira; BATISTA, Nildo Alves. Avaliação da formação interprofissional no ensino superior em saúde: aspectos da colaboração e do trabalho em equipe. Avaliação, Campinas; Sorocaba, v. 16, n. 1, p. 167-184, mar, 2011. Disponível em: <http://www.scielo.br/scielo.php?script=sci_arttext\&pid=S141440772011000100009\&lng=pt\&nrm=iso\&tlng=pt>. Acesso em: 24 maio 2014.

ALMEIDA FILHO, Naomar. Transdisciplinariedade e o paradigma pós-disciplinas em saúde. Saúde e Sociedade, São Paulo, v. 14, n. 3, p. 30-50, set./dez. 2005.

ALMEIDA FILHO, Naomar. Higher education and health care in Brazil. The Lancet, London, UK, v. 377, n. 9781, p. 1898-1900, jun. 2011.

ALMEIDA FILHO, Naomar. Contextos, impasses e desafios na formação de trabalhadores em Saúde Coletiva no Brasil. Ciência \& Saúde Coletiva, Rio de Janeiro, v. 18, n. 6, p. 1677 1682, 2013.

ALMEIDA FILHO, Naomar. Bacharelado Interdisciplinar em Saúde: revolução na educação superior no campo da saúde? In: TEIXEIRA, F. T.; COELHO, M. T. D. (Org.). Uma experiência inovadora no ensino superior: Bacharelado Interdisciplinar em Saúde. Salvador: EDUFBA, 2014. Prefácio, p. 11-2.

ALMEIDA FILHO, Naomar et al. Formação médica na UFSB: I. Bacharelado Interdisciplinar em saúde no primeiro ciclo. Revista Brasileira de Educação Médica, Rio de Janeiro, v. 38, n. 3, p. 337-348, 2014.

ALMEIDA FILHO, Naomar et al. Formação médica na UFSB: II. O desafio da profissionalização no regime de ciclos. Revista Brasileira de Educação Médica, Rio de Janeiro, v. 39, n. 1, p. 123-143, 2015.

AMORETTI, Rogério. A educação médica diante das necessidades sociais em saúde. Revista Brasileira de Educação Médica, Rio de Janeiro, v. 29, n. 2, 136-146, 2005.

AYRES, José Ricardo. Organização das ações de atenção à saúde: modelos e práticas. Saúde e Sociedade, São Paulo, v. 18, sup. 2, p. 11-23, 2009.

BATISTA, Nildo Alves. Educação interprofissional em saúde: concepções e práticas.

Caderno FNEPAS, Rio de Janeiro, v. 2, p. 25-28, 2012.

BRASIL. Lei $n^{\circ}$ 9.394/96 de 20/12/1996. Estabelece as diretrizes e bases para a educação nacional. Diário Oficial da União. Brasília: 23 dez. 1996.

BRASIL. Decreto $n^{\circ}$ 6.096, de 24 de abril de 2007. Institui o Programa de Apoio a Planos de Reestruturação e Expansão das Universidades Federais - REUNI. Diário Oficial da União, Poder Executivo, Brasília, DF, 25 abr. 2007. Seção 1, p. 7. Disponível em:

<http://www.planalto.gov.br/ccivil_03/_Ato2007-2010/2007/Decreto/D6096.htm>. Acesso em: 24 maio 2014.

CANADIAN INTERPROFESSIONAL HEALTH COLLABORATIVE (CIHC). What is... Interprofessional Education (IPE). Fev. 2009. Disponível em:

<http://www.cihc.ca/files/CIHC_Factsheets_IPE_Feb09.pdf>. Acesso em: 10 maio 2016. istep?

CARPENTER, J.; HEWSTONE, M. Shared learning for doctors and social workers:

evaluation of a programme. The British Journal of Social Work, Oxford, v. 26, p. 239-257, 1996. 
A formação em regime de ciclos do Bacharelado Interdisciplinar em Saúde da Universidade Federal da Bahia e a proposta de Educação Interprofissional

COHN, Amélia; ELIAS, Paulo. Saúde no Brasil: políticas e organização de serviços. 5. ed. São Paulo: Cortez/Cedec, 2003.

COOPER, H. et al. Developing an evidence base for interdisciplinary learning: a systematic review. J AdvNurs, Hull, UK, v. 2, p. 228-237, 2001.

CURRAN, V. R.; SHARPE, D. A framework for integrating interprofessional education curriculum in the health sciences. Educ for Health, Urbana, USA, v. 20, n. 3, p. 1-7, 2007.

D'AMOUR, D.; OANDANSAN, I. Interprofessionality as the field of interprofessional practice and interprofessional education: An emerging concept. Journal of Interprofessional Care, London, UK, v. 1, p. 8-20, 2005.

FREETH, D. et al. Effective interprofessional education: development, delivery and evaluation. Oxford: Blackwell Publishing, 2005.

FRENK, J. et al. Health professional for a new century: transforming education to strengthen health systems in an interdependent world. The Lancet, London, UK, v. 376, p. 1923-1958, 2010.

GADELHA, Carlos Augusto Gabrois. O complexo industrial da saúde e a necessidade de um enfoque dinâmico na economia da saúde. Ciência \& Saúde Coletiva, Rio de Janeiro, v. 8, n. 2, p. 521-535, 2003.

GADELHA, Carlos Augusto Gabrois; COSTA, Laís. Saúde e Desenvolvimento no Brasil: Estado da arte e desafios. Rev Saúde Pública, São Paulo, v. 46, n. 1, fev. 2012.

HAMMICK, M. et al. A best evidence systematic review of interprofessional education: BEME Guide n. 9. Medical Teacher, Dundee, UK, v. 29, n. 8, p. 735-751, 2007.

HORAK, B.; O`LEARY, K., L, C. Preparing health care professionals for quality improvement: the George Washington University/George Mason University experience. Quality Management Health Care, London, UK, v. 6, p. 21-30, 1998.

ILLICH, Ivan. A expropriação da Saúde: nêmesis da medicina. 3. ed. Rio de Janeiro: Nova Fronteira, 1975.

LANGTON, H. Interprofessional education in higher education institutions: models, pedagogies and realities. In: BLUTEAU, P.; JACKSON, A. (Eds.). Interprofessional education. Making it Happen. Basingstoke: Palgrave Macmillan, 2009. p. 37-58.

LAPKIN, S.; LEVVETT-JONES, T.; GILLIGAN, C. A systematic review of the effectiveness of interprofessional education in health professional programs. Nurse Education Today, London, UK, v. 33, p. 90-102, 2013.

LEAVISS, J. Exploring the perceived effect of an undergraduate multiprofessional educational intervention. Medical Education, Vancouver, BC, v. 34, p. 483-486, 2000 .

LUECHT, R. M.et al. Assessing professional perceptions: design and validation of an interdisciplinary education perception scales. Journal of Allied Health, Washington DC, v. 19, p. 181-191, 1990.

LUZZI, Daniel Angel; PHILIPPI JR., Arlindo. Interdisciplinaridade, pedagogia e didática da complexidade na formação superior. In: PHILLIPI JR, A.; SILVA NETO, A. J. (Eds.).

Interdisciplinaridade em ciência, tecnologia e inovação. Baureri: Manole, 2011.

MCFADYEN, A.K.; MACLAREN, W.M.; WEBSTER, V. S. The interdisciplinar education perception scale (IEPS): An alternative modeled sub-scale structure and its reliability. J. Interprofessional Care, London, UK v. 21, n. 4, p. 433-443, 2007. 
MATTOS, Ruben. Integralidade, Trabalho, Saúde e Formaçãoprofissional: algumas reflexões críticas feitas com base na defesa de alguns valores. In: MATTA, G. C.; LIMA, J. C. (Orgs.). Estado, sociedade e formação profissional em Saúde. Rio de Janeiro, FIOCRUZ, EPJV, 2008. p. 313-352.

MERHY, Emerson Elias; FEUERWERKER, Laura Camargo Macruz; CECCIM, Ricardo Burg. Educación Permanente en Salud: una estrategia para intervenir en la micropolítica del trabajo en salud. Salud Colectiva, Lanus, Argentina, v. 2, n. 2, p. 147-160, 2006.

MICCAS, Fernanda Lupinno; BATISTA, Sylvia Helena Sousa da Silva. Educação permanente em saúde: metassíntese. Rev. Saúde Pública, São Paulo, v. 48, n. 1, p. 170-185, 2014.

NOGUEIRA, Roberto Passos. A Saúde pelo avesso. Natal, RN, SeminareEditora, 2003.

PARSELL, G.; BLIGH, J. Interprofessional learning. Postgraduate Medical Journal, Hong Kong, China, v. 74, n. 868, p. 89-95, 1998.

PEDUZZI, Marina. Mudanças tecnológicas e seu impacto no processo de trabalho em saúde. Trabalho Educação e Saúde, Rio de Janeiro, v. 1, n. 1, p. 75-91, mar. 2003.

PEDUZZI, Marina. Trabalho e educação na saúde: ampliação da abordagem de recursos humanos. Ciência \& Saúde Coletiva, Rio de Janeiro, v. 18, n. 6, p. 1535-1543, 2013.

PETRIE, Hugh G. Do you see what I see? The epistemology of interdisciplinary inquiry? Journal of Aesthetic Education, Urbana, USA, v. 10, p. 29-43, 1976.

PINTO, Isabela et al. Trabalho e educação em Saúde no Brasil: tendências da produção científica entre 1990-2010. Ciência \& Saúde Coletiva, Rio de Janeiro, v. 18, n. 6, p. 1525 1534, 2013.

REEVES, Scott. Porque precisamos da educação interprofissional para um cuidado efetivo e seguro. Interface, São Paulo, v. 20, n. 56, p. 185-96, 2016.

REEVES, S.; PERRIER, L.; GOLDMAN, J.; FREETH, D.; SWARENSTEIN, M. Interprofessional education: effects on professional practice and health care outcomes. Cochrane Database Syst Rev., London, UK, v. 1, p. 124-125, 2008.

ROCHA, Marcelo Nunes et al. Educação Superior em Saúde: contexto institucional de criação do Bacharelado Interdisciplinar. In: TEIXEIRA, Carmen Fontes; COELHO, Maria Thereza Avila Dantas (Org.). Uma experiência inovadora no ensino superior: bacharelado interdisciplinar em saúde. Salvador: Edufba, 2014, p. 33-52.

ROCHA, Marcelo Nunes et al. Revisão da produção científica brasileira sobre o ensino de graduação em saúde (1974-2011). In: TEIXEIRA, Carmen Fontes; COELHO, Maria Thereza Avila Dantas (Org.) Interdisciplinaridade na educação superior: o bacharelado em saúde. Salvador: EDUFBA, 2016. p. 25-42.

SANTOS, Boaventura de Sousa; ALMEIDA FILHO, Naomar. A universidade no século XXI: para uma Universidade Nova. Coimbra: Almedina, 2012.

SILVA, Rinaldo Henrique Aguilar. Educação interprofissional na graduação em saúde: aspectos avaliativos da implantação na Faculdade de Medicina de Marília (Famema). Educar em Revista, Curitiba, v. 39, p. 159-175, 2011.

TEIXEIRA, Carmen Fontes. Profissionais e trabalhadores de Saúde no Brasil: para onde vamos? Ciência \& Saúde Coletiva, Rio de Janeiro, v. 18, n. 6, p. 1535-1543, 2013. 
A formação em regime de ciclos do Bacharelado Interdisciplinar em Saúde da Universidade Federal da Bahia e a proposta de Educação Interprofissional

TEIXEIRA, Carmen Fontes.; COELHO, Maria Thereza Avila Dantas; ROCHA, Marcelo Nunes Dourado. Bacharelado interdisciplinar: uma proposta inovadora na educação superior em saúde no Brasil. Ciência \& Saúde Coletiva, Rio de Janeiro, v. 18, n. 6, p. 1635-1646, 2013.

TEIXEIRA, Carmen Fontes; COELHO, Maria Thereza Avila Dantas. Processo de implantação do Projeto Político-Pedagógico do BI em saúde 2008-2011: fazendo caminhos ao andar. In: TEIXEIRA, Carmen Fontes; COELHO, Maria Thereza Avila Dantas (Org.). Uma experiência inovadora no ensino superior: Bacharelado Interdisciplinar em Saúde. EDUFBA, 2014. p. 73-107.

THISTLETHWAITE, J. Interprofessional education: a review of context, learning and the research agenda. Medical Education, Vancouver, BC, v. 46, p. 58-70, 2012.

UNIVERSIDADE FEDERAL DA BAHIA (UFBA). Bacharelados Interdisciplinares: projeto pedagógico. Salvador, 2008.

UNIVERSIDADE FEDERAL DA BAHIA (UFBA). Projeto pedagógico dos bacharelados interdisciplinares. Salvador, 2010. Disponível em:

<https://www.ufba.br/sites/devportal.ufba.br/files/implant_reuni.pdf>. Acesso em: 24 maio 2014.

\section{WORLD HEALTH ORGANIZATION. Framework for action on interprofessional education and collaborative practice. Geneva: WHO, 2010.}

WORLD HEALTH ORGANIZATION. Continuing education for physicians. Geneva: WHO, 1988a.

WORLD HEALTH ORGANIZATION. Learning together to work together for health. Geneva: WHO, 1988b.

Renata Meira Veras

${ }^{1}$ Universidade Federal da Bahia | Instituto de Humanidades, Artes e Ciências | Programa de Pós Graduação Estudos Interdisciplinares sobre a Universidade Salvador |BA | Brasil. Contato: renata.veras@ufba.br ORCID (iD) https://orcid.org/0000-0002-1681-1401

Maria Thereza Ávila Dantas Coelho

${ }^{2}$ Universidade Federal da Bahia | Instituto de Humanidades, Artes e Ciências | Programa de Pós Graduação Estudos Interdisciplinares sobre a Universidade Salvador | BA | Brasil. Contato: therezacoelho.ihac@gmail.com ORCID (iD) https://orcid.org/0000-0001-7857-7473

Carmen Fontes Teixeira

${ }^{3}$ Universidade Federal da Bahia | Instituto de Humanidades, Artes e Ciências | Programa de Pós Graduação Estudos Interdisciplinares sobre a Universidade Salvador | BA | Brasil. Contato: carment@ufba.br ORCID (iD) https://orcid.org/0000-0002-8080-9146

Martha Azucena Traverso-Yépez

${ }^{4}$ Memorial University of Newfoundland | Faculty of Medicine | Community Health and Humanities St. John's | Canadá. Contato: mtraverso@mun.ca ORCID (D) https://orcid.org/0000-0002-0224-6093 